\title{
Human recombinant glutamate oxaloacetate transaminase 1 (GOT1) supplemented with oxaloacetate induces a protective effect after cerebral ischemia
}

\author{
M Pérez-Mato ${ }^{1}$, P Ramos-Cabrer ${ }^{1}$, T Sobrino ${ }^{1}$, M Blanco ${ }^{1}$, A Ruban ${ }^{2}$, D Mirelman ${ }^{3}$, P Menendez ${ }^{4,5}$, J Castillo ${ }^{1}$ and F Campos ${ }^{*, 1}$
}

Blood glutamate scavenging is a novel and attractive protecting strategy to reduce the excitotoxic effect of extracellular glutamate released during ischemic brain injury. Glutamate oxaloacetate transaminase 1 (GOT1) activation by means of oxaloacetate administration has been used to reduce the glutamate concentration in the blood. However, the protective effect of the administration of the recombinant GOT1 (rGOT1) enzyme has not been yet addressed in cerebral ischemia. The aim of this study was to analyze the protective effect of an effective dose of oxaloacetate and the human rGOT1 alone and in combination with a non-effective dose of oxaloacetate in an animal model of ischemic stroke. Sixty rats were subjected to a transient middle cerebral artery occlusion (MCAO). Infarct volumes were assessed by magnetic resonance imaging (MRI) before treatment administration, and $24 \mathrm{~h}$ and 7 days after MCAO. Brain glutamate levels were determined by in vivo MR spectroscopy (MRS) during artery occlusion $(80 \mathrm{~min})$ and reperfusion $(180 \mathrm{~min})$. GOT activity and serum glutamate concentration were analyzed during the occlusion and reperfusion period. Somatosensory test was performed at baseline and 7 days after MCAO. The three treatments tested induced a reduction in serum and brain glutamate levels, resulting in a reduction in infarct volume and sensorimotor deficit. Protective effect of rGOT1 supplemented with oxaloacetate at 7 days persists even when treatment was delayed until at least $2 \mathrm{~h}$ after onset of ischemia. In conclusion, our findings indicate that the combination of human rGOT1 with low doses of oxaloacetate seems to be a successful approach for stroke treatment

Cell Death and Disease (2014) 5, e992; doi:10.1038/cddis.2013.507; published online 9 January 2014

Subject Category: Neuroscience

Stroke is a leading cause of mortality and morbidity in developed countries, with increasing incidence because of the progressive aging of the population. Pharmacological or mechanical reperfusion therapy is the most effective treatment during the acute phase of ischemic stroke and it is associated with good outcome in $50-70 \%$ of cases. However, these treatments are only applicable to $<10 \%$ of patients because of the short therapeutic window. ${ }^{1}$ Owing to these therapeutic limitations, the development of new and effective therapies to be used during the acute phase of ischemic stroke is in high demand.

After ischemic stroke, there is a rapid but transient elevation of glutamate into the extracellular space followed by a marked increase in intracellular calcium, which provokes a neuronal death through an excitotoxicity mechanism. ${ }^{2}$ Consequently, calcium and glutamate antagonists have been widely studied as protective agents in experimental models of cerebral ischemia with encouraging results. Unfortunately, they failed or displayed severe adverse effects when they were tested in clinical trials. ${ }^{3}$ Nevertheless, because of the central role of glutamate in the ischemic cascade, the mitigation of glutamate excitotoxicity remains one of the most promising strategies for the development of effective treatments to minimize neurological damage following an acute ischemic stroke. In this sense, novel therapeutic approaches should aim at reducing the increased glutamate levels produced early after the acute phase of ischemia.

Previous studies have shown that a decrease in blood glutamate concentration leads to a larger natural glutamate gradient between the brain and blood, facilitating the efflux of

\footnotetext{
${ }^{1}$ Department of Neurology, Neurovascular Area, Clinical Neurosciences Research Laboratory, Hospital Clínico Universitario, Health Research Institute of Santiago de Compostela (IDIS), University of Santiago de Compostela, Santiago de Compostela, Spain; ${ }^{2}$ Department of Neurobiology, The Weizmann Institute of Science, Rehovot, Israel; ${ }^{3}$ Department of Biological Chemistry, Weizmann Institute of Science, Rehovot, Israel; ${ }^{4}$ Josep Carreras Leukemia Research Institute, Cell Therapy Program of the University of Barcelona, Barcelona, Spain and ${ }^{5}$ Institució Catalana de Recerca i Estudis Avançats (ICREA), Barcelona, Spain

${ }^{*}$ Corresponding author: F Campos, Department of Neurology, Neurovascular Area, Clinical Neurosciences Research Laboratory, Health Research Institute of Santiago de Compostela (IDIS), Hospital Clínico Universitario, University of Santiago de Compostela, c/Travesa da Choupana s/n, 15706 Compostela, Santiago de, Spain. Tel: +34 981 951098; Fax: +34 981 951098; E-mail: francisco.campos.perez@sergas.es

Keywords: animal model; cerebral ischemia; GOT; glutamate; neuroprotection; oxaloacetate

Abbreviations: ADC, apparent diffusion coefficient; AST, aspartate transaminase; GOT1, glutamate oxaloacetate transaminase 1; MCAO, occlusion of the middle cerebral artery; MRI, magnetic resonance imaging; MRS, magnetic resonance spectroscopy; MTT, thiazolyl blue tetrazolium bromide; RF, radiofrequency; rGOT1, recombinant glutamate oxaloacetate transaminase 1; STAIR, stroke therapy academic industry roundtable; STEAM, stimulated-echo acquisition mode; TBI, traumatic brain injury

Received 01.7.13; revised 13.11.13; accepted 18.11.13; Edited by A Verkhratsky
} 
excess extracellular brain glutamate into the blood. ${ }^{4}$ Reduction in glutamate concentration in blood is induced through the activity of the blood-resident enzyme glutamate oxaloacetate transaminase 1 (GOT1), which catalyzes the reversible transformation of oxaloacetate and glutamate to aspartate and $\alpha$-ketoglutarate. Thus, the increase in the co-substrate (oxaloacetate) shifts the equilibrium of the reaction to the right side, thereby decreasing the blood glutamate concentration and lowering the brain glutamate levels (see Teichberg et al. ${ }^{4}$ and Campos et al. ${ }^{5}$ for review).

Based on this mechanism, we ${ }^{6}$ and others ${ }^{4}$ have demonstrated that the intravenous (i.v.) administration of oxaloacetate in a rat model of ischemia induced by transient occlusion of the middle cerebral artery occlusion (MCAO) decreases the glutamate concentration in blood and induces a lowering of infarct volume and edema after ischemia. These effects were also associated with a significant reduction in the sensorimotor deficit. To confirm that the protective effect was mediated by a decrease in the brain glutamate levels, we performed magnetic resonance spectroscopy (MRS) in the infarct region. Spectroscopic analysis revealed that the increase in brain glutamate seen in control animals after MCAO was significantly reduced in animals treated with oxaloacetate. 4,6 These experimental results showed that systemic reduction in glutamate concentration induced by means of exogenous oxaloacetate administration could be used as a novel and efficient protective strategy in ischemic stroke. Importantly, however, the i.v. administration of humans with oxaloacetate has potential serious limitations as the required effective dosage of oxaloacetate in humans, corresponding to that used in rats, might be toxic. ${ }^{7}$ In a previous retrospective study with $>400$ human patients, we have found that ischemic patients with good outcome at 3 months after stroke, showed lower glutamate concentration and higher GOT activity (>18 U/I) in serum. ${ }^{8,9}$ Therefore, it is tentative to postulate that treatments based on the administration of recombinant GOT1 (rGOT1) alone or supplemented with low doses of oxaloacetate could improve the protective effects of oxaloacetate administered on its own.

The purpose of this study was to analyze the protective effect of oxaloacetate and human rGOT1 alone and in combination with a low dose (non-effective dose) of oxaloacetate in the rat model of ischemia induced by transient $\mathrm{MCAO}$, and to compare their effects with a control group treated with saline.

\section{Results}

Animals included in the study. A total of 119 animals were used in this study (Table I). Thirty-six animals were used in the rGOT1 dose-response study, six animals per group (six groups of treatments). In this study, no animal deaths were observed within the first $24 \mathrm{~h}$ after treatments administration. In the protective study, 60 animals were included, six animals/group (four groups in the spectroscopic study and other six groups in the non-spectroscopic study). Eighteen animals were excluded, 16 of them were not included because of unsuccessful MCA occlusion or reperfusion and two animals died $24 \mathrm{~h}$ after surgery (one treated with saline and one treated with rGOT1). Finally, five animals were used as sham-operated control rats (sham) without MCAO.

Serum glutamate concentration is reduced by oxaloacetate and rGOT1 treatments in healthy animals. Administration of saline (control group) did not affect $(P=0.373)$ the basal concentration of glutamate during $24 \mathrm{~h}$ after the i.v. administration (Figure 1a), demonstrating that the surgical procedure did not interfere with the serum glutamate concentration. Injection of glutamate $(15 \mathrm{mM})$ induced a significant $(P<0.05)$ increase in serum glutamate concentration $1 \mathrm{~h}$ after administration (Figure $1 \mathrm{~b}$ ). Basal concentration was normalized $3 \mathrm{~h}$ after treatment administration. This effect shows that the administration of glutamate in healthy animals could be used to simulate the increase in serum glutamate, a hallmark biochemical parameter after ischemia.

On the basis of previous reports where rGOT1 has been used to study other pathologies, ${ }^{10,11}$ we started with a dose of $6.44 \mu \mathrm{g}$ per $100 \mathrm{~g}$ to analyze the capacity of the enzyme to metabolize serum glutamate. Administration of $6.44 \mu \mathrm{g}$ per $100 \mathrm{~g}$ rGOT1 induced a reduction $(P<0.01)$ (Figure $1 \mathrm{c})$ in serum glutamate concentration with respect to the maximum increase observed after glutamate injection. The maximum effect appeared between 2 and $4 \mathrm{~h}$ after administration of treatment. A higher dose of $12.88 \mu \mathrm{g}$ per $100 \mathrm{~g}$ rGOT1 induced a robust reduction $(P<0.01)$ in serum glutamate concentration (Figure 1d). Similar to the previous dose, the maximum lowering effect appeared between 2 and $4 \mathrm{~h}$ after administration of treatment; however, in this case, the capacity to reduce serum glutamate concentration was much higher. Administration of rGOT1 $25.76 \mu \mathrm{g}$ per $100 \mathrm{~g}$ induced a significant $(P=0.007)$ reduction in serum glutamate concentration (Figure 1e). The maximum effect appeared between 2 and $4 \mathrm{~h}$ after administration of the treatment; however, the capacity of this dose to reduce serum glutamate concentration was similar to the $12.88 \mu \mathrm{g}$ per $100 \mathrm{~g}$ dose. These findings showed that $12.88 \mu \mathrm{g}$ per $100 \mathrm{~g}$ seems to be the most appropriate dose of rGOT1 to achieve a significant reduction in the serum glutamate concentration in the animals. Of note, serum glutamate concentrations were normalized 4-6h after rGOT1 administration, demonstrating that the effect of the enzymatic treatment is transient.

In order to determine whether the effect of the enzyme could be potentiated by oxaloacetate (co-substrate of the enzymatic reaction), the dose of rGOT1 (12.88 $\mu \mathrm{g}$ per $100 \mathrm{~g}$ ) was supplemented with a non-effective dose of oxaloacetate $(1.5 \mathrm{mg}$ per $100 \mathrm{~g})$. In our previous study (already published $^{6}$ ), we had described that a dose of $1.5 \mathrm{mg}$ per $100 \mathrm{~g}$ of oxaloacetate did not affect the serum glutamate levels. Similar results were observed again in this study (Figure 1f). Administration of rGOT1 $12.88 \mu \mathrm{g}$ per $100 \mathrm{~g}$ with oxaloacetate $(1.5 \mathrm{mg}$ per $100 \mathrm{~g})$ induced an immediate and significant $(P<0.01)$ lowering of serum glutamate concentration, which is the gold standard approach for reducing increased glutamate levels after brain ischemia. The decrease in glutamate 

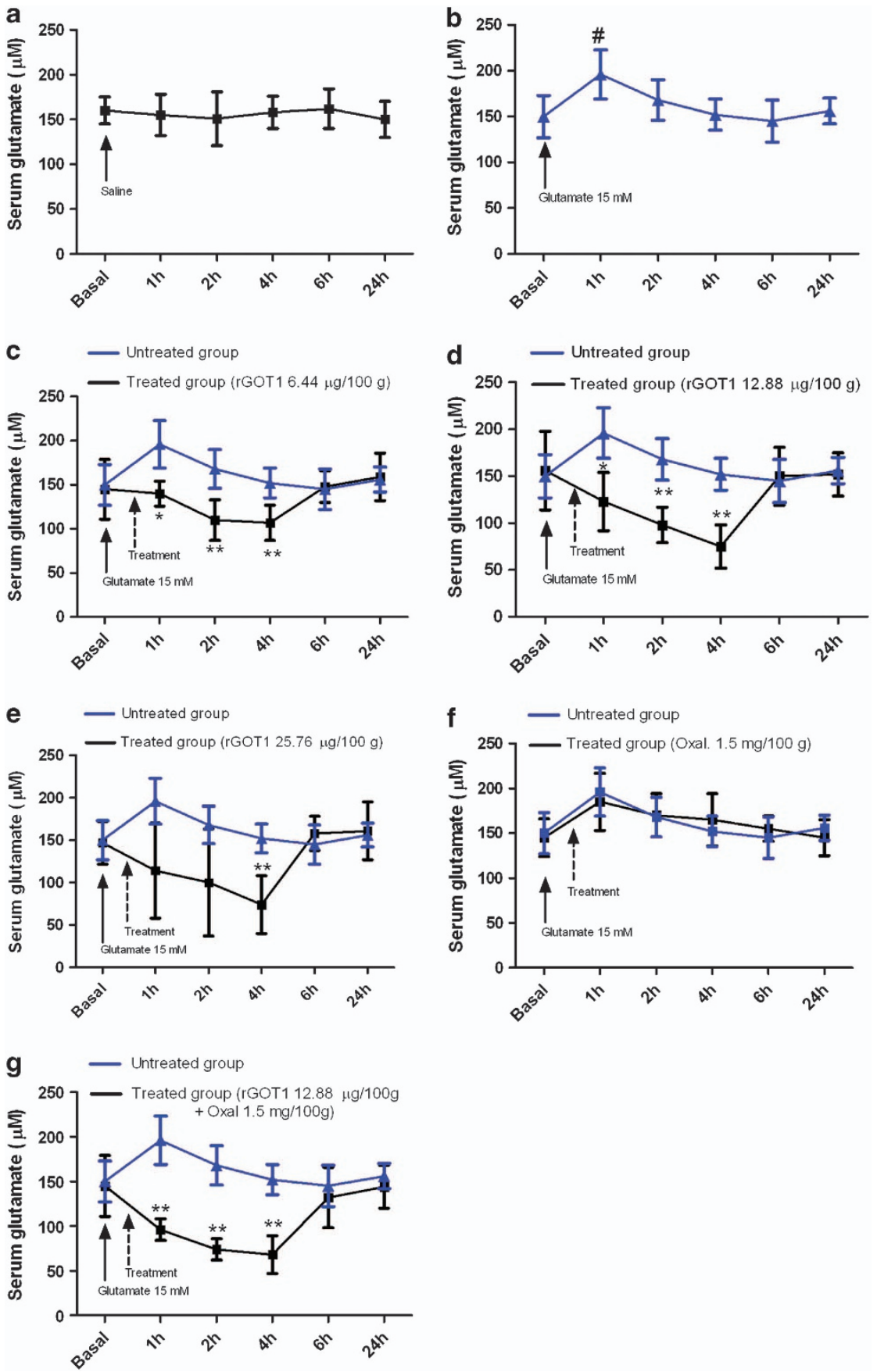

Figure 1 Effect of rGOT and oxaloacetate (Oxal) on serum glutamate levels in healthy rats. Glutamate $15 \mathrm{mM}$ was injected i.v. 30 min prior to treatments. (a) Rats treated with saline (control group). (b) Rats treated with glutamate $15 \mathrm{mM}$. (c) Comparison between rats treated with glutamate $15 \mathrm{mM}$ (blue line) and rats treated with glutamate $15 \mathrm{mM}$ and rGOT1 $6.44 \mu \mathrm{g}$ per $100 \mathrm{~g}$ (black line). (d) Comparison between rats treated with glutamate $15 \mathrm{mM}$ (blue line) and rats treated with glutamate $15 \mathrm{mM}$ and rGOT1 $12.88 \mu \mathrm{g}$ per $100 \mathrm{~g}$ (black line). (e) Comparison between rats treated with glutamate $(15 \mathrm{mM})$ (blue line) and rats treated with glutamate (15 mM) and with rGOT1 (25.76 $\mu \mathrm{g}$ per $100 \mathrm{~g}$ ). (f) Comparison between rats treated with glutamate $15 \mathrm{mM}$ (blue line) and rats treated with glutamate $(15 \mathrm{mM})$ and oxaloacetate (Oxal) $(1.5 \mathrm{mg} \mathrm{per} 100 \mathrm{~g})$. (g) Comparison between rats treated with glutamate $(15 \mathrm{mM})$ (blue line) and rats treated with glutamate $(15 \mathrm{mM})$ and rGOT1 $(12.88 \mu \mathrm{g}$ per $100 \mathrm{~g})$ plus oxaloacetate (Oxal) $(1.5 \mathrm{mg}$ per $100 \mathrm{~g})$. Solid arrows denote the injection of glutamate. Dashed arrows indicate the injection of treatments. Serum samples were taken in basal conditions and 1,2, 4,6 and $24 \mathrm{~h}$ after glutamate injection. Data are shown as mean \pm S.E.M. ${ }^{\#} P<0.05$ compared to the basal levels; ${ }^{*} P<0.05,{ }^{* *} P<0.01$ compared with animals treated with glutamate $15 \mathrm{mM}$

appeared 30 min (with respect to the basal values) after treatment administration and remained during at least $3 \mathrm{~h}$ (Figure 1g); however, no significant differences with respect to rGOT1 $12.88 \mu \mathrm{g}$ pet 100 alone (Figure 1d) were observed.
These results led us to examine whether the protective efficacy of rGOT1 $(12.88 \mu \mathrm{g}$ per $100 \mathrm{~g})$ or rGOT1 $(12.88 \mu \mathrm{g}$ per $100 \mathrm{~g})$ supplemented with oxaloacetate $(1.5 \mathrm{mg}$ per $100 \mathrm{~g})$ in ischemic animals was higher than that observed previously ${ }^{6}$ with oxaloacetate $3.5 \mathrm{mg}$ per $100 \mathrm{~g}$. 
rGOT1 with or without supplementation of oxaloacetate induces a protective effect on ischemic animals. As it was previously shown, ${ }^{6} \mathrm{MCAO}$ induced an increase of $30 \%$ of serum glutamate concentration $1 \mathrm{~h}$ after reperfusion (Figure 2), returning to normal levels $3 \mathrm{~h}$ later. In shamoperated rats in which MCA was not occluded, no changes in plasma glutamate concentration were detected (data not shown). The increase in serum glutamate concentration after cerebral ischemia was inhibited $(P<0.05)$ by each one of the three treatments; rGOT1 (12.88 $\mu \mathrm{g}$ per $100 \mathrm{~g})$, oxaloacetate $(3.5 \mathrm{mg}$ per $100 \mathrm{~g})$ and rGOT1 plus oxaloacetate $(12.88 \mu \mathrm{g}$ per $100 \mathrm{~g}+1.5 \mathrm{mg}$ per $100 \mathrm{~g}$, respectively) (Figure 2). The small differences observed between the treatments were not statistically significant.

To confirm that treatment with rGOT1 induced an increase in systemic GOT1 activity, their levels were measured at different time points (Figure 3). GOT1 activities in the control and oxaloacetate groups were not altered after treatment, whereas rGOT1 treatments induced a significant $(P<0.01)$ increase in serum GOT activity $1 \mathrm{~h}$ after administration, which returned close to normal levels $24 \mathrm{~h}$ after treatment administration.

Quantitative analysis of MRS revealed a persistent increase in brain glutamate levels after the occlusion of MCA in the control group. Glutamate levels in the brain parenchyma were significantly decreased $(P<0.05$ relative to the control animals), with each of the three treatments tested (Figures $4 \mathrm{a}$ and $\mathrm{b}$ ). To demonstrate that the reduction in serum and brain glutamate levels observed after treatment administration leads to a protective effect in our animal model of cerebral ischemia, infarct volumes were measured at $24 \mathrm{~h}$ and 7 days after ischemia and were compared with the control group. Figure 5a shows that, although all treatments were able to induce a significant $(P<0.05)$ protection against the ischemic damage both $24 \mathrm{~h}$ and 7 days after the onset of ischemia, rGOT1+ oxaloacetate revealed to be the more effective treatment ( $P<0.01$, with respect to the control group). Analysis of infarct volumes adjusted to the ipsilateral hemisphere showed a similar protective profile (data not shown). Infarct volumes measured in those animals subjected to the spectroscopy protocol (Figure 5b) showed the same protective results for the three treatments. Diffusion images revealed that all animals subjected to spectroscopy presented the same ischemic damage before the treatment administration.

To determine the therapeutic window of rGOT1 treatments, two groups of animals were treated $1 \mathrm{~h}$ after reperfusion (140 min after occlusion). Analysis of serum glutamate concentration in animals treated with rGOT1 $(12.88 \mu \mathrm{g}$ per $100 \mathrm{~g})$ or rGOT1 plus oxaloacetate $(12.88 \mu \mathrm{g}$ per $100 \mathrm{~g}+1.5 \mathrm{mg}$ per $100 \mathrm{~g}$, respectively) showed that both treatments caused a significant $(P<0.05)$ inhibition of the increase in glutamate observed in the control group (Figure 6). Analysis of infarct volume determined that both treatments displayed similar infarct volume reduction at $24 \mathrm{~h}$ when administered immediately after reperfusion (Figures $7 \mathrm{a}$ and $\mathrm{b}$ ), however, while the protective effect induced for rGOT1 disappears when this treatment is given $1 \mathrm{~h}$ after reperfusion on day 7; when rGOT1 is administered in

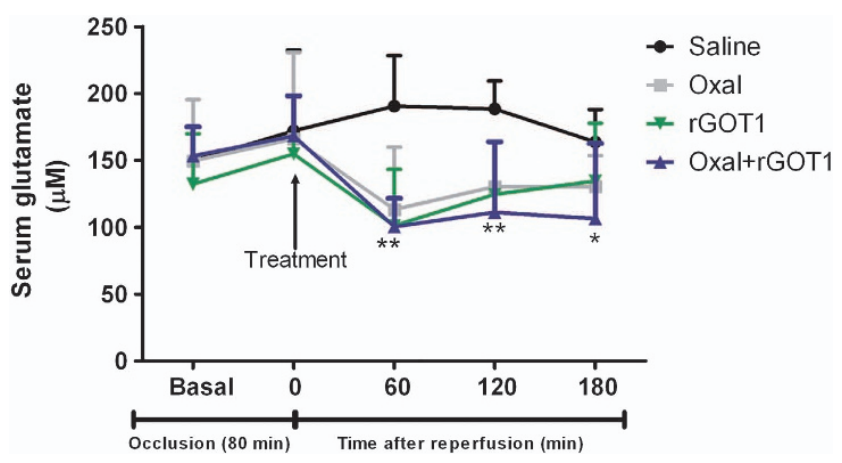

Figure 2 Time course of serum glutamate concentration $(\mu \mathrm{M})$ in MCAO rats. MCAO rats were treated with saline (control group) $(n=6)$, oxaloacetate (Oxal) $3.5 \mathrm{mg}$ per $100 \mathrm{~g}(n=6)$, rGOT1 $12.88 \mu \mathrm{g}$ per $100 \mathrm{~g}(n=6)$ and rGOT1 $12.88 \mu \mathrm{g}$ per $100 \mathrm{~g}$ plus oxaloacetate $1.5 \mathrm{mg}$ per $100 \mathrm{~g}(n=6)$. Solid arrow indicates the time of treatment injection, administered at the moment of reperfusion $(80 \mathrm{~min}$ after occlusion). Serum glutamate concentration was measured under basal conditions (before surgery), at the moment of the reperfusion (before treatment administration), and 1,2 and $3 \mathrm{~h}$ after reperfusion. Data are shown as mean \pm S.E.M. ${ }^{*} P<0.05$, ${ }^{\star \star} P<0.01$ compared with control group

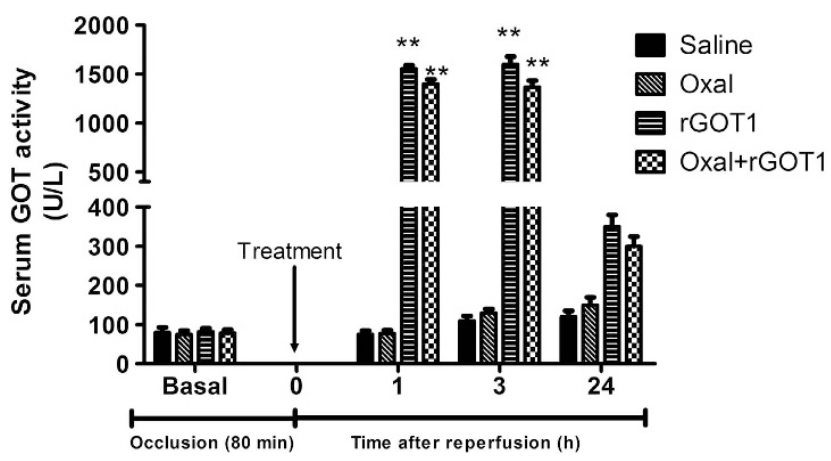

Figure 3 Time course of serum GOT activity in MCAO rats. MCAO rats were treated with saline (control group) $(n=6)$, oxaloacetate (Oxal) $3.5 \mathrm{mg}$ per $100 \mathrm{~g}$ $(n=6)$, rGOT1 $12.88 \mu \mathrm{g}$ per $100 \mathrm{~g}(n=6)$ and rGOT1 $12.88 \mu \mathrm{g}$ per $100 \mathrm{~g}$ plus oxaloacetate $1.5 \mathrm{mg}$ per $100 \mathrm{~g}(n=6)$. Treatments were administered at the moment of reperfusion ( $80 \mathrm{~min}$ after occlusion). GOT activity was measured under basal conditions (before surgery), and 1, 3 and $24 \mathrm{~h}$ after reperfusion. Data are shown as percentage compared with basal levels \pm S.E.M. ${ }^{* *} P<0.01$ with respect to control group

combination with oxaloacetate, the effect persists beyond $1 \mathrm{~h}$ after reperfusion $(P<0.05$, with respect to the control).

Somatosensory test, an important end point assay of drug screening in stroke, confirmed that reduction in infarct volume observed with each of the three treatments was associated with a better neurological outcome measured 7 days after ischemia, showing the treatment rGOT1 + oxaloacetate to be a superior effect $(P<0.01$, with respect to the control) (Figure 8).

In vitro tests for neurotoxicity of oxaloacetate. To determine the potential toxicity of oxaloacetate treatment used in the study, a neuronal culture was exposed to a range of concentrations of oxaloacetate (100 nM to $1 \mathrm{mM})$ during $48 \mathrm{~h}$. Our analysis showed that, under these experimental conditions, this compound did not affect the viability of the neurons (Supplementary Figure I). 

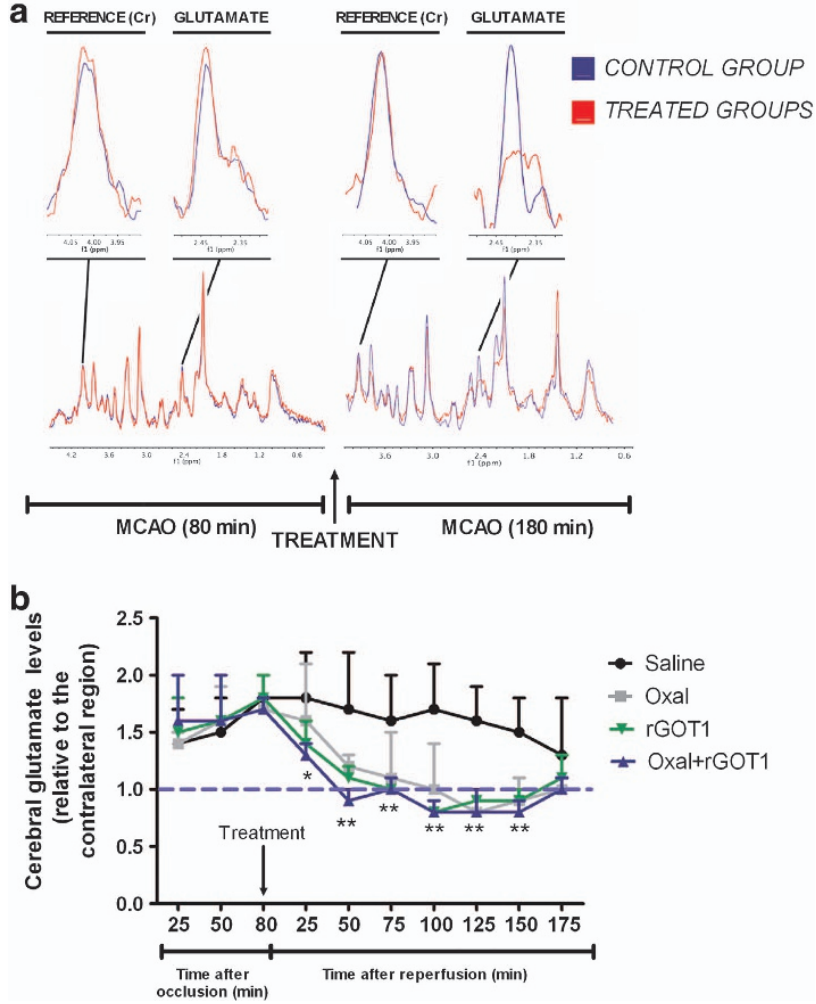

Figure 4 MRS analysis. (a) Quantitative analysis of glutamate signals were normalized to creatine $(\mathrm{Cr})$ peaks for each single spectra. Brain glutamate levels were analyzed during the occlusion ( $80 \mathrm{~min}$ ) and during reperfusion (180 min). Brain glutamate levels were similar in treated (before treatment) and control (treated with saline) ischemic animals during occlusion. However, during reperfusion glutamate levels were lower in treated animals compared with the control. (b) Time course of brain glutamate levels in MCAO rats treated with saline (control group) $(n=6)$, oxaloacetate (Oxal) $3.5 \mathrm{mg}$ per $100 \mathrm{~g}(n=6)$, rGOT1 $12.88 \mu \mathrm{g}$ per $100 \mathrm{~g}(n=6)$ and rGOT1 $12.88 \mu \mathrm{g}$ per $100 \mathrm{~g}$ plus oxaloacetate $1.5 \mathrm{mg}$ per $100 \mathrm{~g}(n=6)$. Solid arrow indicates the moment of i.v treatment. Cerebral glutamate levels were measured during occlusion $(80 \mathrm{~min}$ ) and reperfusion $(180 \mathrm{~min})$. MRS analysis was performed in an independent group of animals. Data are shown as the mean of cerebral glutamate levels relative to the contralateral region \pm S.E.M. Blue dashed line indicates the levels of brain glutamate in the contralateral region. ${ }^{*} P<0.05$, ${ }^{\star \star} P<0.01$ compared with control group

\section{Discussion}

Nowadays, the concept of blood/brain glutamate scavenging is well recognized as a novel and attractive protective strategy to reduce the excitotoxic effect of excess extracellular glutamate that accumulates in the brain following an ischemic stroke. The efficacy of this strategy has been demonstrated in different types of ischemic animal models, and it has also been tested in other pathologies associated with an increase in brain glutamate levels, such as traumatic brain injury (TBI) ${ }^{4}$ or glioma ${ }^{12}$ with successful results. GOT activation by means of exogenous administration of oxaloacetate has been used as the most common approach to reduce the serum glutamate concentration. In this study, we found that the i.v. administration of rGOT1 was also able to induce a reduction in serum and brain glutamate levels, which also resulted in a significant reduction in the infarct volume and improvement in the sensorimotor deficit after ischemia. These effects were similar to those observed with the higher doses of oxaloacetate. ${ }^{6}$ Importantly, the protective effect of rGOT1 was more evident when it was administered in combination with a low concentration of oxaloacetate that had no effect by itself, indicating that the combined administration of rGOT1 and oxaloacetate could be an efficient serum glutamate-scavenging approach. The i.v. administration of an endogenous serum enzyme such as GOT1 as a new protective treatment against ischemia is an interesting strategy because it is unlikely that the administration of rGOT1 can induce toxic effects in humans as the levels of this enzyme varies among healthy human subjects $(7-45 \mathrm{U} / \mathrm{l})$, and has been shown to increase $>10$-fold in patients with liver damage. ${ }^{13}$ On the other hand, the increase in the enzyme activity after the treatment is not much longer than $24 \mathrm{~h}$, and serum glutamate concentrations come back to normal within the first $6 \mathrm{~h}$, which reduces long-term potential adverse effects. However, the effective dose of GOT (12.88 $\mu \mathrm{g}$ per $100 \mathrm{~g}$ ) used in the animals cannot be extrapolated to humans, as GOT levels in rats are almost 10-fold higher than those in humans $(10 \mathrm{U} / \mathrm{l}$ in humans versus $95 \mathrm{U} / \mathrm{I}$ in rats). In this regard, further (pre)-clinical studies based on the administration of GOT should be conducted in humans to determine the effective therapeutic dose of GOT able to induce a significant reduction in serum glutamate in humans. In line with this, we have previously observed that stroke patients who had high GOT activity (average of $17 \mathrm{U} / \mathrm{l}$ ) at admission had reduced serum glutamate concentrations and a significantly better neurological outcome than patients with lower average activity of GOT of $11 \mathrm{U} / \mathrm{I}^{8,9}$ Therefore, it can be hypothesized that the administration of rGOT1 doses able to increase the systemic GOT activity, at least two or three-fold, are necessary for clinical studies in stroke patients.

Regarding the use of oxaloacetate as a serum glutamatescavenging treatment, such a treatment could present some limitations associated with the high dose likely to be necessary in patients to achieve the same effects than those observed in experimental animals. ${ }^{7}$ However, although to date there are no consistent data in humans confirming this toxic effect, a study published in the 60 's,${ }^{14}$ in which oxaloacetate was used to treat diabetic patients (from 200 to $1000 \mathrm{mg}$ per day in three divided doses given orally) did not report any effect on liver function tests and blood acetone and cholesterol levels at the doses administered. Oral administration is not comparable to i.v. administration used in this study; nevertheless, this clinical study represents evidence for the relative safety of the administration of this molecule in humans. In this line, we also observed that oxaloacetate does not affect the viability of the neuronal culture, which is used as a current assay for neurotoxicity. ${ }^{10}$ The protective effects of oxaloacetate have also been discussed. ${ }^{5}$ As this molecule participates in the energy metabolism and provides an antioxidant protection of cells subjected to stress, such as hydrogen peroxide, it has been suggested that the protective role of oxaloacetate could be related to other mechanisms different from serum glutamate reduction. Studies in a TBI model showed that the protective effect of oxaloacetate was abolished in the presence of excess glutamate in serum or when it was administered in combination with maleate (a GOT inhibitor) ${ }^{15}$ which effectively demonstrates its serum glutamate-scavenging action. 
a
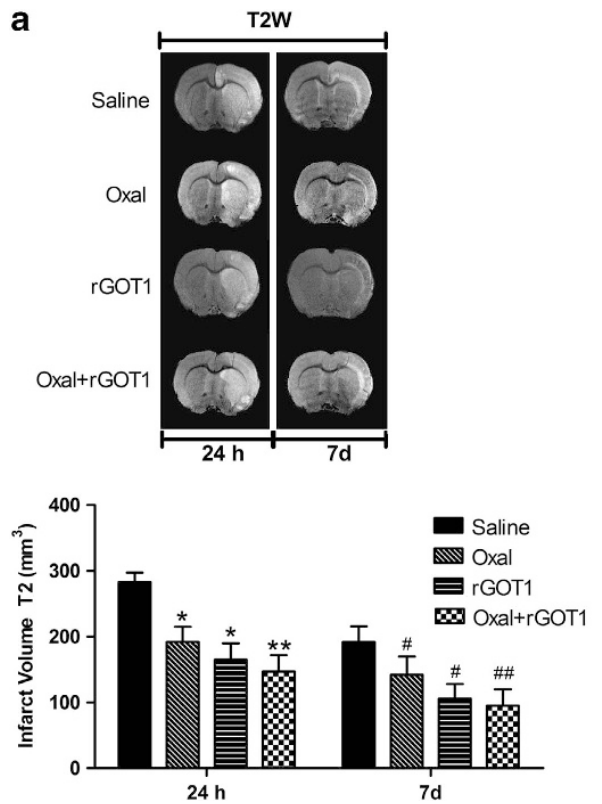

b
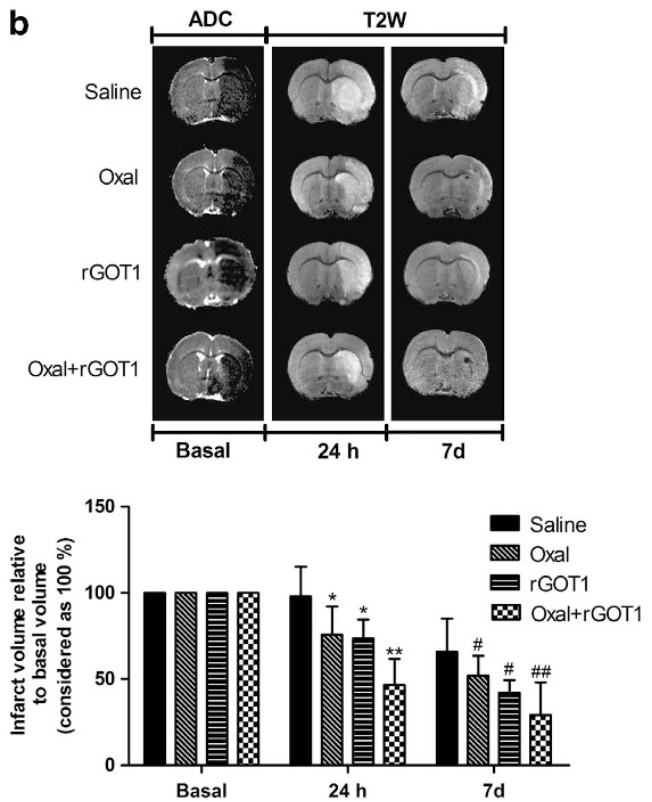

Figure 5 Infarct size assessed by means of MRI in MCAO rats. MCAO rats were treated with saline (control group), oxaloacetate (Oxal) $3.5 \mathrm{mg} \mathrm{per} 100 \mathrm{~g}$, rGOT1 $12.88 \mu \mathrm{g}$ per $100 \mathrm{~g}$ and rGOT1 $12.88 \mu \mathrm{g}$ per $100 \mathrm{~g}$ plus oxaloacetate $1.5 \mathrm{mg}$ per $100 \mathrm{~g}$. Treatments were administered at the moment of the reperfusion. Infarct size was assessed in ischemic rats not subjected to spectrocopy analysis (a) as well as in ischemic rats subjected to spectroscopy analysis (b). Infarct sizes were measured at $24 \mathrm{~h}$ and $7 \mathrm{days}$ after ischemia. Only those animals subjected to spectroscopy analysis presented ADC basal volumes (determined before the administration of treatment). Data are shown as the mean of infarct volume $\left(\mathrm{mm}^{3}\right) \pm$ S.E.M in a, and percentage relative to the basal volume in $\mathbf{b}$. ${ }^{\star} P<0.05,{ }^{* *} P<0.01$ with respect to the control group at $24 \mathrm{~h}$; ${ }^{\#} P<0.05$, $\#$ \# $<0.01$ as well as on day 7

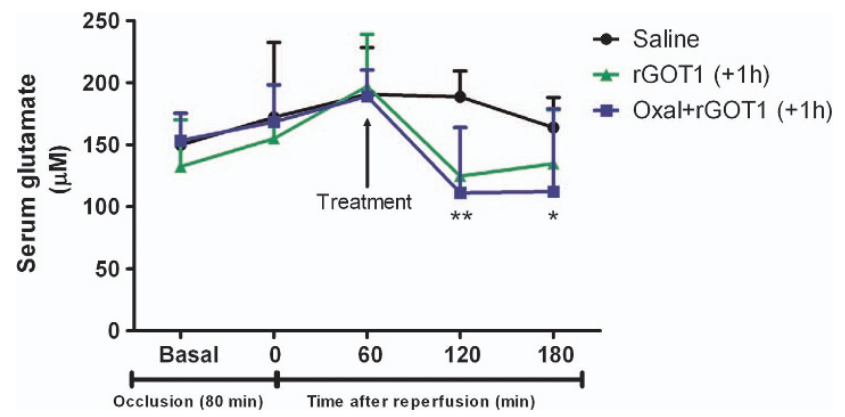

Figure 6 Time course of serum glutamate concentration $(\mu \mathrm{M})$ in MCAO rats. MCAO rats were treated with saline (control group) $(n=6)$, rGOT1 $12.88 \mu \mathrm{g}$ per $100 \mathrm{~g}(n=6)$ and rGOT1 $12.88 \mu \mathrm{g}$ per $100 \mathrm{~g}$ plus oxaloacetate $1.5 \mathrm{mg}$ per $100 \mathrm{~g}$ $(n=6) 1 \mathrm{~h}$ after reperfusion. Solid arrow indicates the time of the i.v. treatments. Serum glutamate concentration was measured under basal conditions (before surgery), at the time of reperfusion (before treatment administration), and 1, 2 and $3 \mathrm{~h}$ after reperfusion. Data are shown as mean \pm S.E.M. ${ }^{*} P<0.05,{ }^{* *} P<0.01$ with respect to the control group

Interestingly, the protective effect of rGOT1 $(12.88 \mu \mathrm{g}$ per $100 \mathrm{~g}$ ) was potentiated when it was administered in combination with a non-effective low dose of oxaloacetate $(1.5 \mathrm{mg}$ per $100 \mathrm{~g})$ but not with higher doses of the enzyme $(25.76 \mu \mathrm{g}$ per $100 \mathrm{~g}$ ). These findings are in agreement with a previous glioblastoma study reporting that the combination of rGOT1 with low amounts of oxaloacetate together with temozolodine significantly extends the lifespan of tumor-bearing animals. ${ }^{12}$ The therapeutic synergistic effect observed between oxaloacetate and GOT can be attributed to the fact that the endogenous oxaloacetate concentration can become a limiting factor of the enzymatic reaction when GOT activity is increased after treatment. Therefore, attending to the rapid and maintained reduction in serum glutamate concentration, treatments based on the combined administration of rGOT1 supplemented with a low concentration of oxaloacetate could be optimal to reach the maximum protective effect in ischemia. Of note, the low dose of oxaloacetate is sufficient to increase the glutamate-scavenging activity of rGOT1, which reduces the potential complications associated with a high dose of this molecule.

It is crucial to develop treatment approaches capable of overcoming the narrow therapeutic window in stroke in order to mitigate the detrimental effects of the excess of brain glutamate after ischemia, which represents a stumbling-block from a clinical point of view. Owing to this limitation, only those treatments (mainly, NMDA antagonists) with protective effects beyond the first $1-2 \mathrm{~h}$ after the onset of ischemia have shown clinical interest. ${ }^{3}$ In our ischemic experimental model, we have observed that brain and serum glutamate levels appear to be increased in the first $2 \mathrm{~h}$ after reperfusion. We observed that lowering of glutamate by means of GOT treatments had protective effects even when the treatment was delayed until $1 \mathrm{~h}$ after reperfusion. Further experiments need to be carried out to determine the length of the therapeutic window; however, based on our results, it may be speculated that the therapeutic window for the administration of rGOT1 could be up to $2 \mathrm{~h}$ after the onset of ischemia. As this treatment does not require a prior computerized tomography scan, it could be given as early as possible, perhaps even as ambulatory treatment suggesting potential clinical application. In comparison with previous glutamate antagonists, another important advantage of 
a
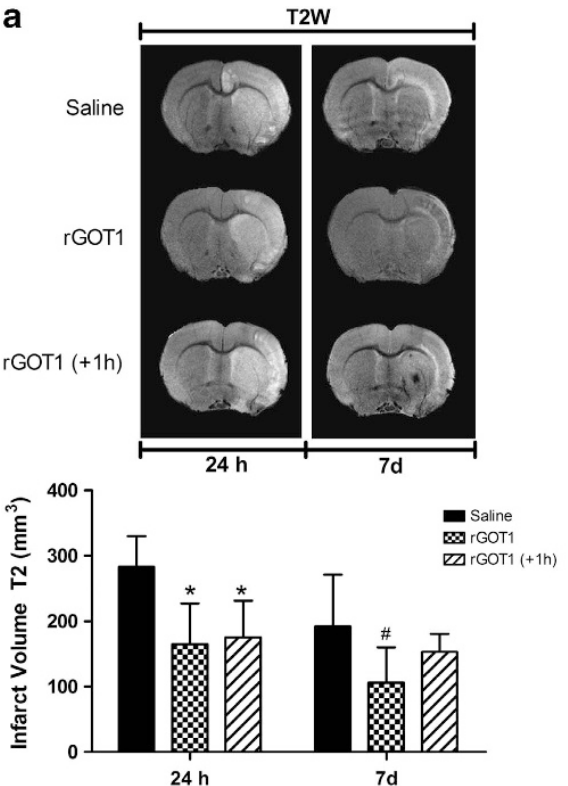

b
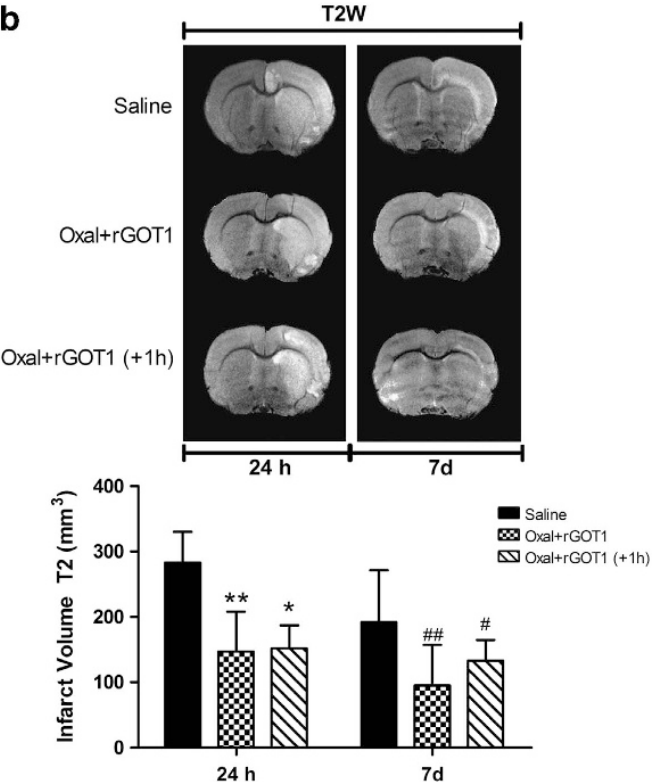

Figure 7 Infarct size assessed by means of MRI in MCAO rats. MCAO rats were treated at two different times. (a) saline (control group) ( $n=6)$, rGOT1 12.88 $\mu \mathrm{g}$ per $100 \mathrm{~g}$ (rGOT1) ( $n=6$ ) at the moment of reperfusion, and rGOT1 $12.88 \mu \mathrm{g}$ per $100 \mathrm{~g}$ (rGOT1 $+1 \mathrm{~h})(n=6) 1 \mathrm{~h}$ after reperfusion. (b) Saline (control group) ( $n=6)$, rGOT1 $12.88 \mu \mathrm{g}$ per $100 \mathrm{~g}$ plus oxaloacetate $1.5 \mathrm{mg}$ per $100 \mathrm{~g}$ (Oxal + rGOT1) $(n=6)$ at the time of the reperfusion and rGOT1 $12.88 \mu \mathrm{g}$ per $100 \mathrm{~g}$ plus oxaloacetate $1.5 \mathrm{mg}$ per $100 \mathrm{~g}(\mathrm{Oxal}+\mathrm{rGOT} 1+1 \mathrm{~h})(\mathrm{n}=6) 1 \mathrm{~h}$ after reperfusion. Data are shown as the mean of infarct volume $\left(\mathrm{mm}^{3}\right) \pm$ S.E.M. ${ }^{*} P<0.05,{ }^{* *} P<0.01$ compared with control group at $24 \mathrm{~h} ;{ }^{\#} P<0.05,{ }^{\#} P<0.01$ with respect to control group at day 7

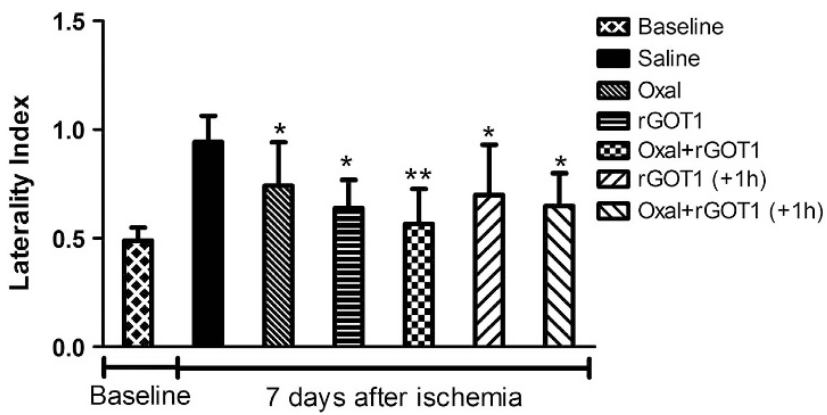

Figure 8 Effect of treatments on somatosensory test. Sensorimotor deficits after an ischemic insult were assessed using the cylinder test and quantified by laterality index. $+1 \mathrm{~h}$ denotes that those treatments were administered $1 \mathrm{~h}$ after reperfusion. Other treatments were administered at the moment of the reperfusion. Somatosensory tests were performed 1 day before surgery (baseline) and on day 7 after MCAO. Values represent the mean \pm S.E.M, $n=6$. ${ }^{\star} P<0.05,{ }^{* \star} P<0.01$ relative to control group

serum-scavenging-based treatments is that their effects are not mediated through the neuronal ionotropic glutamate receptors, thereby avoiding problems of poor blood-brain barrier permeability and potential neurotoxic effects.

In conclusion, in this study we show that oxaloacetate potentiates the protective effect of intravenously administered rGOT1 after ischemia. This effect is mediated through a reduction in serum and brain glutamate levels. The protective effect was observed even when the treatments were initiated as soon as $2 \mathrm{~h}$ after the onset of ischemia. The robust protection shown by the combination of rGOT1 and low doses of oxaloacetate proposes that this strategy may represent a valid approach for a successful acute-phase stroke treatment.

\section{Materials and Methods}

Animals. Experimental protocols were approved by the local Animal Care Committee according to the European Union (EU) rules (86/609/CEE, 2003/65/CE and 2010/63/EU). Male Sprague-Dawley rats (Harlan Laboratories, Barcelona, Spain) with a weight of $250-300 \mathrm{~g}$ were used. Rats were watered and fed $\mathrm{ad}$ libitum. Anesthesia was induced by inhalation of $5 \%$ sevoflurane in a nitrous oxide/ oxygen mixture (70/30). Rectal temperature was maintained at $37 \pm 0.5^{\circ} \mathrm{C}$ by using a feedback-controlled heating pad. Glucose levels were analyzed before surgery (ranging from 180 to $220 \mathrm{mg} / \mathrm{dl}$ ).

Surgical procedures. Transient focal ischemia was induced in rats by using the transient MCAO following the surgical procedures previously described. ${ }^{6}$ In brief, under an operating microscope, the left common, external and internal carotid arteries were dissected from connective tissue through a midline neck incision. The left external carotid artery and pterygopalatine artery of the internal carotid artery were separated and ligated by $5-0$ silk sutures. A 23-mm segment of 3-0 nylon monofilament suture with the tip rounded by heat was inserted into the stump of the left common carotid artery and advanced into the internal carotid artery to $20 \mathrm{~mm}$ from the bifurcation to occlude the origin of the MCA. The suture was removed after $80 \mathrm{~min}$ of occlusion. A laser-Doppler flow probe (tip diameter $1 \mathrm{~mm}$ ) attached to a flowmeter (PeriFlux 5000; Perimed AB, Stockholm, Sweden) was located over the thinned skull in the MCA territory ( $4 \mathrm{~mm}$ lateral to bregma) to obtain a continuous measure of relative cerebral flow during the experiment. Only animals with a cerebral serum flow reduction of over $70 \%$ and with reperfusion after occlusion were included in the study. Experimental procedures were performed following five criteria derived from the Stroke Therapy Academic Industry Roundtable (STAIR) group guidelines for preclinical evaluation of stroke therapeutics: ${ }^{11,16}$ (1) cerebral serum flow was measured to confirm the vascular occlusion as an index of the reliability of the ischemic model; (2) animals were randomly assigned to treatment groups of the study; (3) researchers were blinded to treatment administration; (4) researchers were blinded to treatments during outcome assessment; and (5) temperature was controlled during the ischemic period.

Experimental protocol. In our previous study, we demonstrated that an i.v. dose of $3.5 \mathrm{mg}$ per $100 \mathrm{~g}$ (weight of animal) of oxaloacetate induced a significant reduction in serum glutamate concentration, whereas a dose $1.5 \mathrm{mg}$ per $100 \mathrm{~g}$ 
(low dose) was found to be ineffective. ${ }^{6}$ However, as a dose-response study on the effect of the i.v. administration of rGOT1 on serum glutamate concentration has not been yet tested, we divided our study into two steps: (A) a rGOT1 doseresponse study and (B) protective study.

(A) rGOT1 dose-response study: here, healthy rats were first subjected to an artificial increase in serum glutamate concentration to determine the effective dose of rGOT1 able to induce a reduction in glutamate concentration ( $n=6$ per dose). The artificial increase in serum glutamate concentration was induced by means of the administration of glutamate $15 \mathrm{mM}(1 \mathrm{ml}$ per $300 \mathrm{~g}$ weight of the animal). This procedure was used to mimic the increase in serum glutamate observed after an ischemic insult. Glutamate $15 \mathrm{mM}$ was injected at the beginning of the experiment (after animal anesthesia induction), and 30 min later rGOT1 was given. Serum samples $(500 \mu l)$ were taken (from vein tail) under basal conditions (before glutamate injection), and 1, 2, 4, 6 and $24 \mathrm{~h}$ after glutamate injection. Treatments were administered through the jugular vein in a bolus form. All rGOT1 doses were dissolved in saline $(0.9 \%$ of $\mathrm{NaCl})$ and the concentration was adjusted to inject $1 \mathrm{ml}$ per animal. pH was adjusted to 7.4. Pure human rGOT1 (5800 U/l, analyzed with Reflotron GOT (aspartate transaminase, AST) Test) was provided by Professor David Mirelman from Weizmann Institute, Israel.

(B) Protective study: in this phase, the protective effects of oxaloacetate $3.5 \mathrm{mg}$ per $100 \mathrm{~g}$ (weight of animal) and the optimal doses selected in rGOT1 doseresponse study were tested in an ischemic model. To determine the protective effect of the treatments, brain and serum glutamate levels, serum GOT activity, infarct volume and functional deficit were measured in the experimental animals. Treatments were administered immediately after reperfusion $(80 \mathrm{~min}$ after occlusion) or $1 \mathrm{~h}$ after reperfusion (140 min after occlusion).

Brain glutamate levels were measured during occlusion $(80 \mathrm{~min})$ and during reperfusion (180 $\mathrm{min}$ ) using the MRS technique. Serum glutamate concentration was determined under basal conditions (before surgery), immediately after reperfusion ( $80 \mathrm{~min}$ after occlusion) and 1,2 and $3 \mathrm{~h}$ after reperfusion. GOT activity was measured under basal conditions (before surgery) and 1, 3 and $24 \mathrm{~h}$ after reperfusion. As it was not technically accurate to measure the serum and brain glutamate levels after reperfusion at the same time in the same animal, treatments were tested in two independent groups of animals ( $n=6$ each). One group was used to measure brain glutamate levels and the other to determine the serum glutamate concentration and GOT activity. Infarct volume was determined during occlusion (only in those animals subjected to spectroscopy analysis), $24 \mathrm{~h}$ and 7 days after ischemia. Sensorimotor test was performed under basal conditions (1 day before surgery) and 7 days after ischemia.

Serum glutamate analysis. Serum samples were collected in test tubes, centrifuged at 3000 r.p.m. for $7 \mathrm{~min}$, serum was removed and immediately frozen and stored at $-80^{\circ} \mathrm{C}$. Serum glutamate concentration was determined by means of Glutamate Assay Kit (Abnova, Taipei City, Taiwan) following the manufacturer's technical specifications.

Serum GOT activity analysis. GOT activity was determined by means of Reflotron GOT (AST) Test following the manufacturer's technical specifications (Roche, Basel, Switzerland).

Magnetic resonance imaging protocol. Infarct size was assessed by means of magnetic resonance imaging (MRI). MRI studies were conducted on a 9.4-T horizontal bore magnet (Bruker BioSpin, Ettligen, Germany) with $12 \mathrm{~cm}$ wide actively shielded gradient coils $(440 \mathrm{mT} / \mathrm{m})$. Radiofrequency (RF) transmission was achieved with a birdcage volume redsonator; signal was detected using a fourelement arrayed surface coil, positioned over the head of the animal, which was fixed with a teeth bar, earplugs and adhesive tape. Transmission and reception coils were actively decoupled from each other. Gradient-echo pilot scans were performed at the beginning of each imaging session for accurate positioning of the animal inside the magnet bore. Apparent diffusion coefficient (ADC) maps were acquired during MCA occlusion ( $80 \mathrm{~min}$ after the onset of ischemia) using a spin-echo echo-planar imaging sequence with the following acquisition parameters: field-of-view $19.2 \times 19.2 \mathrm{~mm}^{2}$, image matrix $128 \times 128$ (in-plane resolution $0.15 \mathrm{~mm} /$ pixel), 14 consecutive slices of $1 \mathrm{~mm}$ thickness, repetition time $=4 \mathrm{~s}$, echo time $=30 \mathrm{~ms}$ and diffusion b values: $0,100,300,600,800,1000$ and $1400 \mathrm{~s} / \mathrm{mm}^{2}$. T2-weighted image was acquired $24 \mathrm{~h}$ and 7 days after the onset of ischemia. All images were processed and maps were constructed with ImageJ (Rasband WS, ImageJ, US National Institutes of Health, Bethesda, MD, USA, http://rsb.info.nih. gov/ij/, 1997-2009).
In vivo MRS. MRS was acquired as previously described. ${ }^{17,18}$ Local shimming was performed by manual adjustment of first- and second-order shim coil currents using a proton-stimulated-echo acquisition mode (STEAM)-waterline sequence. The field homogeneity in a 3- $\mathrm{mm}^{3}$ voxel typically resulted in signal line widths of $10-20 \mathrm{~Hz}$ for the water signal. Water signal was suppressed by variable power RF pulses with optimized relaxation delays (VAPOR). In vivo $1 \mathrm{H}$ magnetic resonance spectra of the rat brain were acquired by using a STEAM sequence with echo time $=3 \mathrm{~ms}$, mixing time $(\mathrm{TM})=5 \mathrm{~ms}$, repetition time $=2500 \mathrm{~ms}, 176$ averages, cubic voxel $=3 \times 3 \times 3 \mathrm{~mm}^{3}(27 \mu \mathrm{l})$. Spectra were processed using MNOVA 7 (Mestrelab Research, Santiago de Compostela, Spain). For the quantitative analysis, glutamate signals were normalized to the creatine peak areas for each single spectrum (Supplementary Figure II). MRS was acquired during the occlusion (80 $\mathrm{min}$ ) and during $180 \mathrm{~min}$ after reperfusion.

Image analysis. All images were processed using ImageJ (Rasband WS, ImageJ, NIH, http://rsb.info.nih.gov/ij). Infarct volumes were determined from (ADC maps) and T2-weighted images by manually selecting areas of reduced $A D C$ values or hyperintense $T 2$ signal by a researcher blinded to the animal protocols.

Sensorimotor test. Sensorimotor deficits after ischemic insult were assessed using the cylinder test as previously described. ${ }^{19}$ This test consists of evaluation of asymmetry of limbs during the exploratory activity. For this test, the animal was put in a cylinder of transparent base of $20 \mathrm{~cm}$ diameter. A video camera is located under this transparent cylinder for recording the vertical exploratory movement of the animal with anterior limbs during 2-10 min, depending on movement grade. For recording analysis, the VirtualDub software was used. Analyzed behaviors were as follows: number of times that the animal touches the cylinder wall and independent use of each limb in contact with the cylinder wall in each upward movement. Laterality index was calculated (the number of times that the animal touches the cylinder with the right leg during the ascendant movement by the number of times that the animals touch with each leg). This index is close to 0.5 for healthy animals and tends to be 0 or 1 for animals that have a preferential use of the left or the right paw, respectively. Somatosensory tests were performed 1 day before MCAO and on day 7 after ischemia during the darkness cycle.

Primary culture of rat cortical neurons. Primary cultures of cortical neurons were performed as described previously. ${ }^{20}$ Brains were removed from fetal Sprague-Dawley rats (Harlan Laboratories, Barcelona, Spain) on embryonic day 18 , and the cortical area was dissected. Neurons were mechanically dissociated in incubation medium consisting of $80 \%$ Eagle's minimum essential medium (MEM) containing $0.6 \%$ glucose, $0.029 \%$ glutamine, $16 \mathrm{mg} / \mathrm{l}$ gentamicin, $10 \%$ horse serum and $10 \%$ fetal calf serum. The dissociated neurons were plated at a density of 100000 cells per well in poly-lysine-precoated 24-multiwell plates. Medium was replaced twice weekly and studies were performed at in vitro on day 8 , when the cultures consisted of $\geq 95 \%$ neurons.

Neuronal toxicity MMT assay. After 8 days in culture, neurons were exposed to different concentrations of oxaloacetate. Cell death was quantified with thiazolyl blue tetrazolium bromide (MTT) (Sigma, St. Louis, MO, USA) $48 \mathrm{~h}$ later. Neurons were incubated in $200 \mu \mathrm{g} / \mathrm{ml} \mathrm{MTT}$ at $37^{\circ} \mathrm{C}$, and after $2 \mathrm{~h}$, the culture medium was aspirated and cells were lysed in $250 \mu \mathrm{l}$ DMSO. Color intensity was measured at $570 \mathrm{~nm}$. Results were expressed as the percentage of absorbance of control wells. Vehicle treatment was used in control group and DMSO $2 \%$ treatment for control death.

Statistical analysis. Results were expressed as mean \pm S.E.M. Statistical analyses were performed using one-way ANOVA followed by a Bonferroni post hoc analysis to determine between-group differences. A $P$-value $<0.05$ was considered as statistically significant. The statistical analysis was conducted using PASW Statistics 18 for Mac (SPSS Inc., Chicago, CA, USA).

\section{Conflict of Interest}

The authors declare no conflict of interest. 
Acknowledgements. This project has been partially supported by grants from the Spanish Ministry of Economy and Competitiveness SAF2011-30517, Xunta de Galicia (Consellería Economía Industria: 10PXIB918282PR; and Consellería Educación: CN2011/010), Instituto de Salud Carlos III (PI11/00909 and CP12/ 03121), Spanish Research Network on Cerebrovascular Diseases RETICSINVICTUS (RD12/0014), Fundación Mútua Madrileña and by the European Union program FEDER. Furthermore, T. Sobrino and P. Ramos-Cabrer are recipients of a research contract from Miguel Servet Program of Instituto de Salud Carlos III. Sponsors did not participate in study design, collection, analysis and interpretation of the data, writing the report or in the decision to submit the paper for publication. P Menendez as an ICREA Research Professor of the Generalitat of Catalunya supported by ISCIII (PI10/00449 and PI12/03112).

\section{Author contributions}

MPM, PRC, TM and JA conduced the experiments. MB, DM, JC and FC analyzed the data. DM, JC and FC designed the study. MB and PM helped in the interpretation of the data. All authors participated in the writing and editing of the manuscript. JC and FC were the principal investigators and supervised the project.

1. Tomsick TA, Khatri $P$, Jovin T, Demaerschalk B, Malisch $T$, Demchuk A et al. Equipoise among recanalization strategies. Neurology 2010; 74: 1069-1076.

2. Lipton P. Ischemic cell death in brain neurons. Physiol Rev 1999; 79: 1431-1568.

3. Ginsberg MD. Neuroprotection for ischemic stroke: past present and future Neuropharmacology 2008; 55: 363-389.

4. Teichberg VI, Cohen-Kashi-Malina K, Cooper I, Zlotnik A. Homeostasis of glutamate in brain fluids: an accelerated brain-to-blood efflux of excess glutamate is produced by blood glutamate scavenging and offers protection from neuropathologies. Neuroscience 2009; 158: 301-308.

5. Campos F, Sobrino T, Ramos-Cabrer P, Castillo J. Oxaloacetate: a novel neuroprotective for acute ischemic stroke. Int J Biochem Cell Biol 2012; 44: 262-265.

6. Campos F, Sobrino T, Ramos-Cabrer P, Argibay B, Agulla J, Perez-Mato M et al. Neuroprotection by glutamate oxaloacetate transaminase in ischemic stroke: an experimental study. J Cereb Blood Flow Metab 2011; 31: 1378-1386.

7. Leibowitz A, Boyko M, Shapira $Y$, Zlotnik A. Blood glutamate scavenging: insight into neuroprotection. Int J Mol Sci 2012; 13: 10041-10066.

8. Campos F, Rodriguez-Yanez M, Castellanos M, Arias S, Perez-Mato M, Sobrino T et al. Blood levels of glutamate oxaloacetate transaminase are more strongly associated with good outcome in acute ischaemic stroke than glutamate pyruvate transaminase levels. Clin Sci (Lond) 2011; 121: 11-17.
9. Campos F, Sobrino T, Ramos-Cabrer P, Castellanos M, Blanco M, Rodriguez-Yanez M et al. High blood glutamate oxaloacetate transaminase levels are associated with good functional outcome in acute ischemic stroke. J Cereb Blood Flow Metab 2011; 31: 1387-1393.

10. Llorens J, Li AA, Ceccatelli S, Sunol C. Strategies and tools for preventing neurotoxicity: to test, to predict and how to do it. Neurotoxicology 2012; 33: 796-804.

11. Stroke Therapy Academic Industry Roundtable (STAIR). Recommendations for standards regarding preclinical neuroprotective and restorative drug development. Stroke 1999; 30: 2752-2758.

12. Ruban A, Berkutzki T, Cooper I, Mohar B, Teichberg VI. Blood glutamate scavengers prolong the survival of rats and mice with brain-implanted gliomas. Invest New Drugs 2012; 30: 2226-2235.

13. Tian Z, Liu H, Su X, Fang Z, Dong Z, Yu C et al. Role of elevated liver transaminase levels in the diagnosis of liver injury after blunt abdominal trauma. Exp Ther Med 2012; 4: 255-260.

14. Yoshikawa K. Studies on the anti-diabetic effect of sodium oxaloacetate. Tohoku J Exp Med 1968; 96: 127-141.

15. Zlotnik A, Gruenbaum SE, Artru AA, Rozet I, Dubilet M, Tkachov S et al. The neuroprotective effects of oxaloacetate in closed head injury in rats is mediated by its blood glutamate scavenging activity: evidence from the use of maleate. J Neurosurg Anesthesiol 2009; 21: 235-241.

16. Philip M, Benatar M, Fisher M, Savitz SI. Methodological quality of animal studies of neuroprotective agents currently in phase II/III acute ischemic stroke trials. Stroke 2009; 40: $577-581$.

17. Higuchi T, Graham SH, Fernandez EJ, Rooney WD, Gaspary HL, Weiner MW et al. Effects of severe global ischemia on $\mathrm{N}$-acetylaspartate and other metabolites in the rat brain. Magn Reson Med 1997; 37: 851-857.

18. Tkac I, Starcuk Z, Choi IY, Gruetter R. In vivo $1 \mathrm{H}$ NMR spectroscopy of rat brain at $1 \mathrm{~ms}$ echo time. Magn Reson Med 1999; 41: 649-656.

19. Schallert T, Fleming SM, Leasure JL, Tillerson JL, Bland ST. CNS plasticity and assessment of forelimb sensorimotor outcome in unilateral rat models of stroke, cortical ablation, parkinsonism and spinal cord injury. Neuropharmacology 2000; 39: 777-787.

20. Moro MA, Fernandez-Tome P, Leza JC, Lorenzo P, Lizasoain I. Neuronal death induced by SIN-1 in the presence of superoxide dismutase: protection by cyclic GMP. Neuropharmacology 1998; 37: 1071-1079.

(2) ()ㅇ Cell Death and Disease is an open-access journal published by Nature Publishing Group. This work is licensed under a Creative Commons Attribution-NonCommercialNoDerivs 3.0 Unported License. To view a copy of this license, visit http://creativecommons.org/licenses/by-nc-nd/3.0/

Supplementary Information accompanies this paper on Cell Death and Disease website (http://www.nature.com/cddis) 